\title{
The Importance of Sub-Nanosecond Fluctuations on the Toughness of Polycarbonate Glasses
}

Christopher L. Soles ${ }^{1,{ }^{*}}$, Adam B. Burns ${ }^{1,+}$, Kanae Ito ${ }^{1,++}$, Edwin Chan ${ }^{1}$, Jianwei Liu ${ }^{2,++\dagger}$, Albert F. Yee ${ }^{3}$, Madhu Sudan Tyagi ${ }^{4}$

(1) NIST Materials Science and Engineering Division, 100 Bureau Drive, Gaithersburg MD; (2) Macromolecular Science and Engineering, University of Michigan, Ann Arbor, MI; (3) Department of Chemical and Biological Engineering, University of California, Irvine, CA; (4) NIST Center for Neutron Research, 100 Bureau Drive, Gaithersburg, MD. 


\section{Supplemental Information}

As discussed in the main text related to Table 1, a suppression of $\left\langle u^{2}\right\rangle_{\text {excess }}$ in the anharmonic regime can be interpreted as an effective stiffening of the effective spring constant for the collective dynamics. In Figure S1, the values of $\langle k\rangle_{\mathrm{AH}}$ are plotted as a function of $\left\langle u^{2}\right\rangle_{\text {excess }}$ across for of the series of PC samples studied here. This graphical representation shows the direct correlation between a stiff effective spring constant and a reduced anharmonicity in the collective dynamics. The correlations in Figure 4 of the main text relating impact strength to $\left\langle u^{2}\right\rangle_{\text {excess }}$ could easily be recast in terms of $\langle k\rangle_{A H}$; a softer effective anharmonic spring constant correlates with a tougher glass.

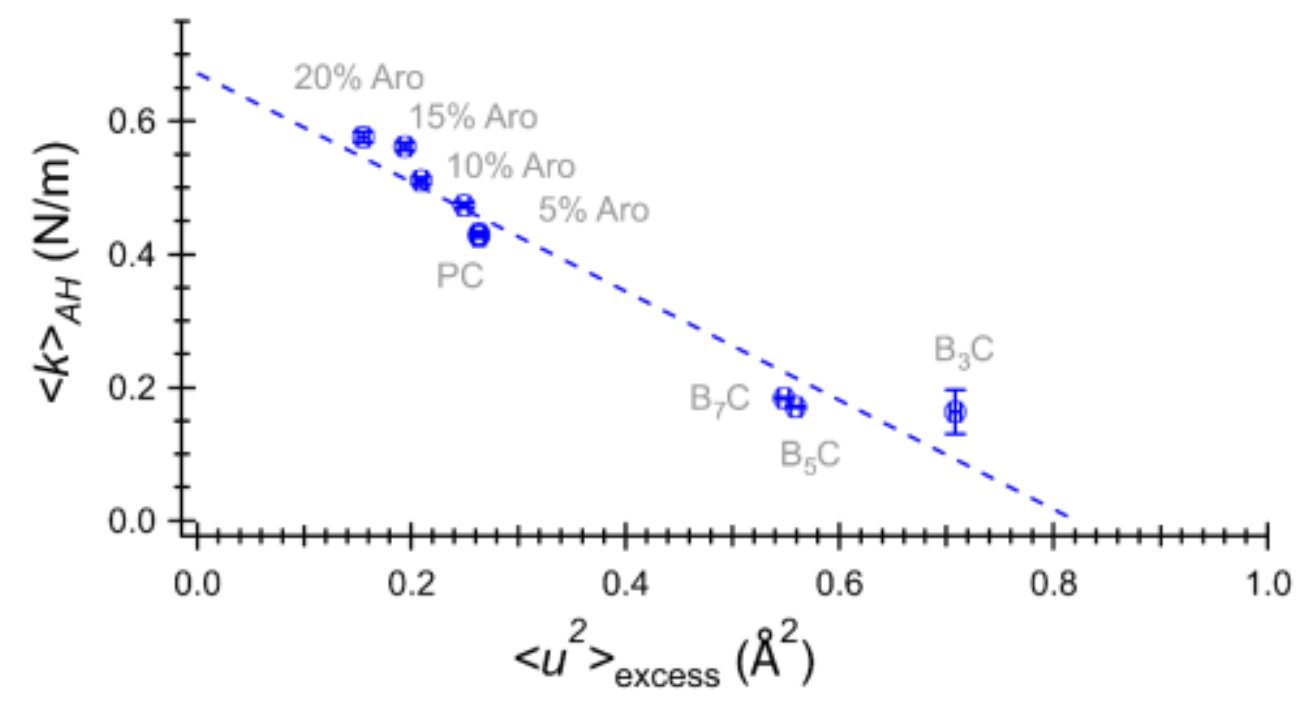

Figure S1. The correlation of $\langle k\rangle_{A H}$ and $\left\langle u^{2}\right\rangle_{\text {excess }}$ for the polycarbonate samples reported in Table 1 of the main text. 


\section{Activation Volume of Yielding Information}
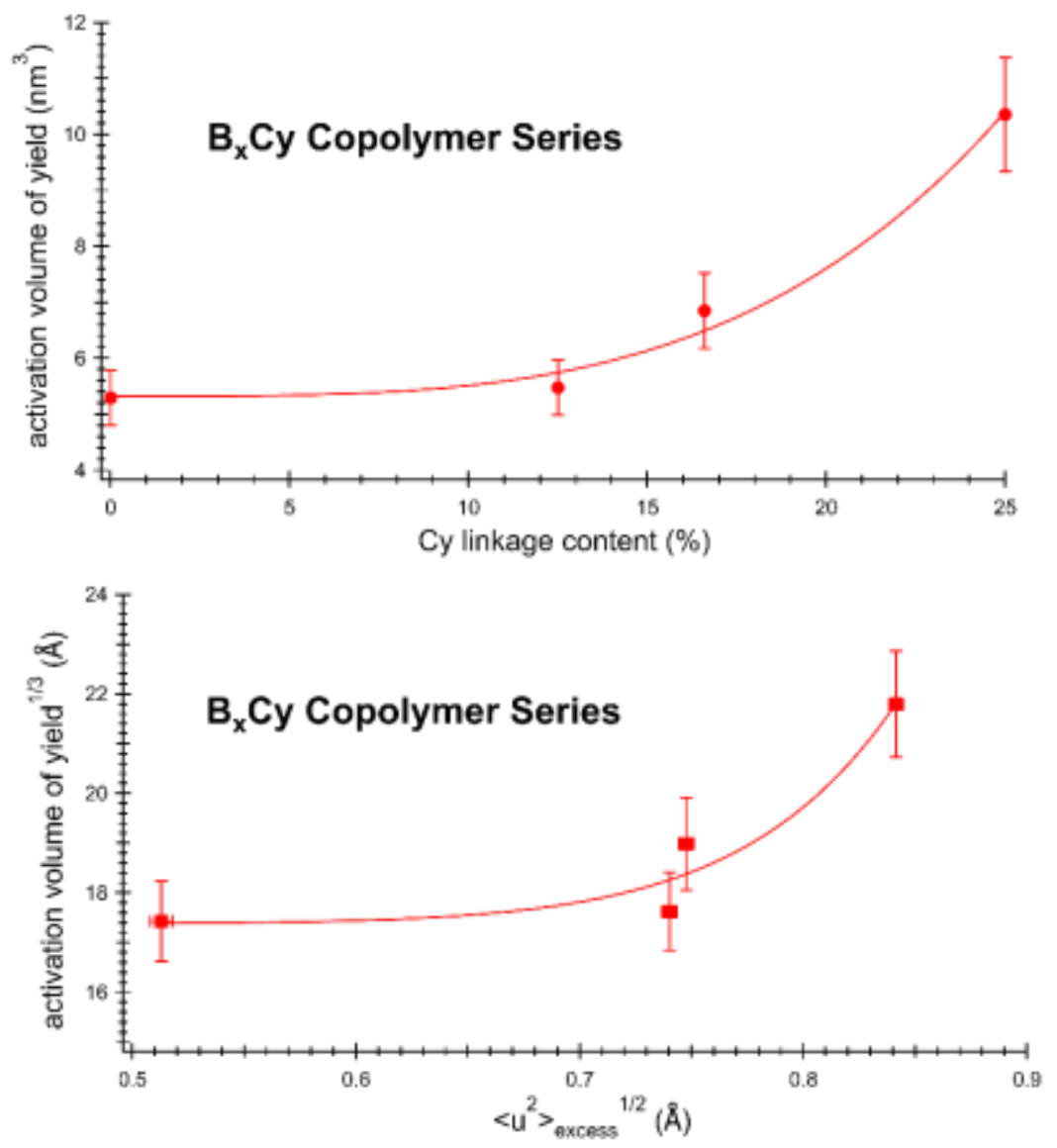

Figure S2. These figures plot the activation volume of the yield process according to an Eyring model of yielding for the $B_{x} C y$ copolymer, as reported in a previous publication. In the upper panel, the activation volume of yield in units of cubic nanometers is plotted as a function of Cy content, showing that the yield process becomes more diffuse as the Cy content increases. The same data in recast in the lower panel where the cube root of the activation volume has been plotted, in units of angstroms, against the square root of $\left\langle u^{2}\right\rangle_{\text {excess, }}$ also in the units of angstroms. The two parameters are within an order of magnitude. This crude comparison is done to make the point that it is reasonable to think about $\left\langle u^{2}\right\rangle_{\text {excess }}$ when we think about yield mechanisms. 